\title{
Computer aided design and analysis on distributors in DAC columns
}

\author{
Chen-Jiann Lin ${ }^{1,}$, Tseng-Hsiang Tse ${ }^{1}$, Liu-Cheng Che ${ }^{1}$, and Liang-Ming Tsai ${ }^{2}$ \\ ${ }^{1}$ Department of Mechanical and Automation Engineering, I-Shou University, 84001 Kaohsiung City, \\ Taiwan \\ ${ }^{2}$ Department of Chemical Engineering, I-Shou University, 84001 Kaohsiung City, Taiwan
}

\begin{abstract}
Dynamic axial compression (DAC) columns are key elements in simulated moving bed, which is a chromatography process in drug industry and chemical engineering. In this study, rules for designing distributors are proposed based on mass conservation and validated by experiments, the computer aided design (CAD) and the computational fluid dynamics (CFD). Experimental works are conducted to choose feasible numerical parameters for simulations. In CFD, the transient laminar flow fields are governed by the momentum and species transport equations with Darcy's law to model the porous zone in the packed bed. Results show that CFD combined with CAD solid modelling is a good approach to explore detailed flow fields in DAC columns and carry out parameter analysis for innovative designs. For further testing and evaluation, a new model of compound distributor is designed, 3D printed and processed in factory for practical applications in preparative chromatography.
\end{abstract}

\section{Introduction}

Simulated moving bed (SMB) chromatography process, which is a multicolumn chromatography process, has been applied to various industrial fields, such as foods, petrochemicals, fine chemicals, and pharmaceuticals [1]. The counter current movement of the adsorbent bed is simulated by periodically shifting the inlet and outlet nodes to the direction of the flow during each switching period. Therefore, the SMB process can achieve high productivity and low solvent consumption [2] and allows significantly higher yield and purity compared to a batch chromatography [3, 4].

Generally, the performance of chromatography depends on the combined effects of DAC column dynamics, the pre and post column geometry [5]. In the beginning of the column, an even fluid distribution must be achieved cross-sectionally through careful design in order to give satisfactory separation efficiency $[6,7]$. Therefore, the distributor at the column inlet will be vital to provide plug flow, hence improve the column efficiency. At the same time appropriate packing materials have to be chosen and the column has to be carefully packed to maintain the flow pattern as close as possible to a plug flow $[8,9]$. The above mentioned factors are especially important to columns with large diameter. If these factors were not considered, band distortion and unsymmetrical peaks would be observed because flow misdistribution can result from faulty header design $[7,10]$. Therefore, DAC *Corresponding author: james88@isu.edu.tw 
columns tend to be of low length over diameter (L/D) for high-productivity considerations, which results in poor flow distributions and reduces the column efficiency. In DAC columns, the distributor (collector) plays a key role to make the plug flow in columns. Manufacturers in this field have been devoted to improving the distributor's performance to raise the efficiency of their products from DAC columns [11, 12].

CFD has well been known for more than 20 years as a pragmatic and reliable tool to describe local hydrodynamics in various separation processes [13]. Its modelling has been applied to investigate the transport phenomena in the preparative chromatography column [14-16]. Therefore, it is proposed to characterize in detailed hydrodynamics of DAC columns based on the recent advances of flow characterisation by CFD, and then to deduce numerical treatments that can be beneficial to simulations. In the present study, CFD is used to access the internal information inside the DAC column and the flow is considered to be laminar due to low flow speed in the micro-particle packed column [9, 17].

For the present research, we present the governing equations for the physical problem and introduce CAD and CFD techniques to simulate flow fields in the DAC column in section 2. Rules to design distributors at the header of DAC columns are also proposed in this section. Results and discussions are stated in section 3, which includes experiments, numerical treatments for CFD, CFD code validations and parameter analysis to investigate flow phenomena. Furthermore, a model of compound distributor is displayed and the prototype is 3D-printed, which is referred to develop DAC columns with low $\mathrm{L} / \mathrm{D}$ for future practical applications. Remarks are concluded in the final section, which includes the present contributions and future works.

\section{Methodology}

The present simulation is simplified as an incompressible laminar flow of a single species that does not generate heat. Inside the DAC column, non-slip boundary conditions are used for the walls and the packed bed is viewed as a porous medium in which the flow of a Newtonian fluid is known to follow Darcy's law at a low Reynolds number. The flowchart for the design of DAC columns in this study is illustrated in Fig. 1.

\subsection{Governing equations}

\subsubsection{Equations in physical domain}

The transient conservation equation for transport of a scalar quantity $\phi$ is demonstrated by the following equation written in integral form for an arbitrary control volume $V$ as follows [18]:

$$
\frac{\mathrm{d}}{\mathrm{dt}} \oint_{\mathrm{V}} \rho \phi \mathrm{dV}+\oint \rho \phi \overrightarrow{\mathrm{V}} \cdot \mathrm{d} \overrightarrow{\mathrm{A}}=\oint \Gamma \nabla \phi \cdot \mathrm{d} \overrightarrow{\mathrm{A}}+\int_{\mathrm{V}} \mathrm{S}_{\phi} \mathrm{dV}
$$

where $\rho$ indicates density; $\overrightarrow{\mathrm{V}}$ is velocity vector; $\overrightarrow{\mathrm{A}}$ is surface area vector; $\mathrm{V}$ is volume; $\Gamma$ is the diffusion coefficient for $\phi$ and $\mathrm{S}_{\phi}$ is source term of $\phi$ per unit volume. The symbol $\phi$ can be replaced by 1 for the continuity equation, and $\mathrm{u}, \mathrm{v}$ and $\mathrm{w}$ are the momentum equations in the $\mathrm{X}, \mathrm{Y}$ and $\mathrm{Z}$ directions, respectively. 


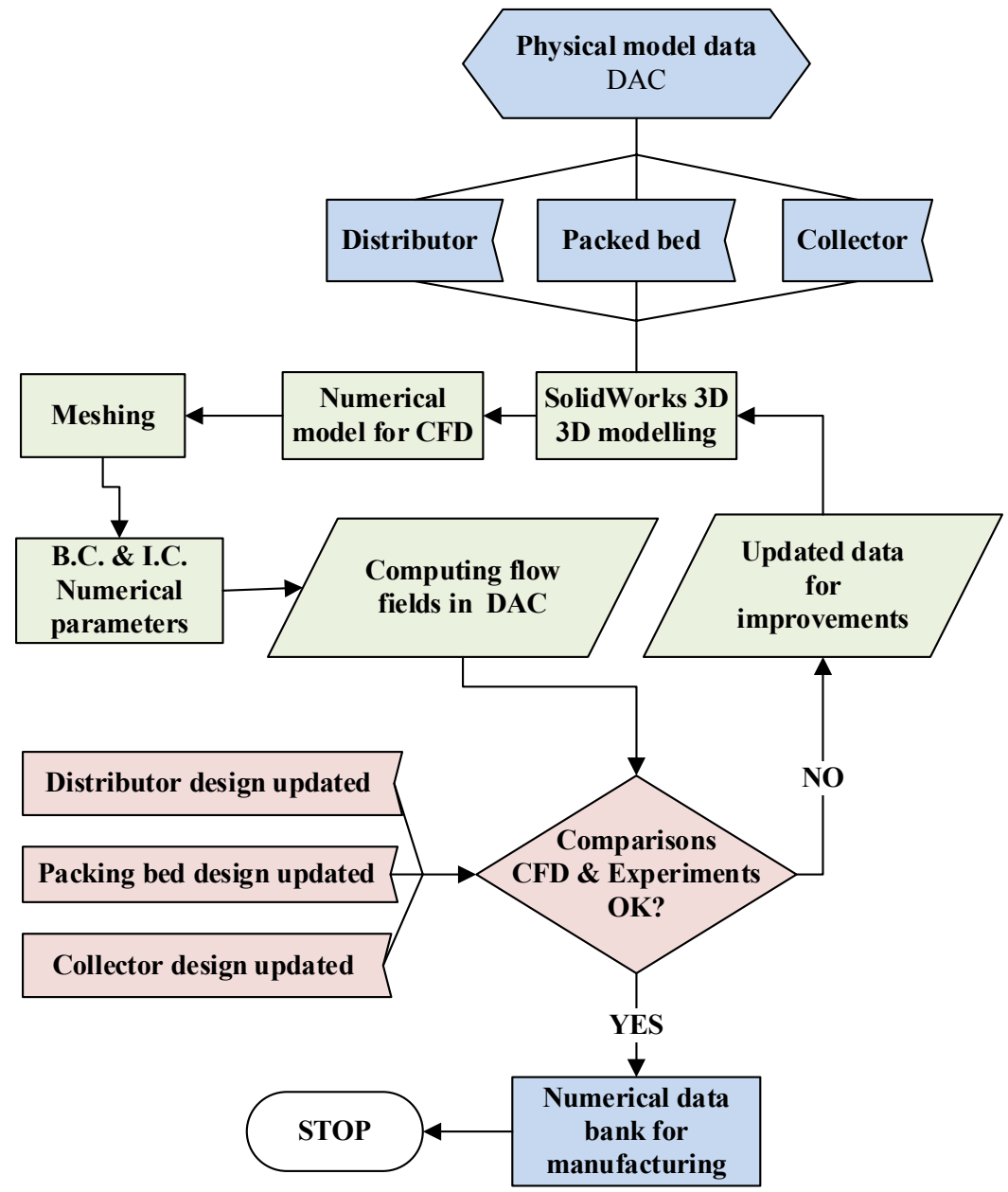

Fig. 1. The flowchart for the design of DAC columns.

Inside the DAC column, the packed bed can be simulated by porous medium, which is expressed as extra source terms including viscous loss (Darcy's law) and inertial loss. The added source terms to model the porous effect is expressed as

$$
\mathrm{S}_{\mathrm{j}}=-\left(\frac{\mu}{\alpha} \mathrm{v}_{\mathrm{j}}+\mathrm{C}^{\prime} \frac{1}{2} \rho|\mathrm{v}| \mathrm{v}_{\mathrm{j}}\right)
$$

where $\alpha$ is permeability ( $1 / \alpha$ is viscous resistance); $\mathrm{C}^{\prime}$ indicate the inertial resistance factor and $|v|$ is the magnitude of the velocity. If the fluid flows very slowly in porous zone for some cases, the inertial loss can be ignored to save the computing time.

In the present work the pulse input was applied and the injected sample was dilute solution. No adsorption between the solute and the stationary phase takes place. Therefore the mass loss between the liquid phase and solid phase could be neglected concerning momentum balance. The species concentration conservation used in present calculation is

$$
\frac{\mathrm{d}}{\mathrm{dt}} \oint_{\mathrm{V}} \rho \mathrm{C}_{\mathrm{i}} \mathrm{dV}+\oint \rho \mathrm{C}_{\mathrm{i}} \overrightarrow{\mathrm{V}} \cdot \mathrm{d} \overrightarrow{\mathrm{A}}=\oint \mathrm{D}_{\mathrm{i}} \nabla \mathrm{C}_{\mathrm{i}} \cdot \mathrm{d} \overrightarrow{\mathrm{A}}+\int_{\mathrm{V}} \mathrm{S}_{\mathrm{C}} \mathrm{dV}
$$


where $C_{i}$ indicates the mass fraction for species $i ; D_{i}$ is the mass diffusion coefficient for species $i$ in the mixture.

\subsubsection{Equations in computational domain}

Since the fluid used in this study is IPA (Isopropyl alcohol), the density is assumed to be constant in the flow field. Equation (1) can be expressed in generalized coordinates using the finite volume method approach for simulation. The discretized formulation for each cell (control volume) based on Eq. (1) yields

$$
\sum_{\mathrm{f}}^{\mathrm{N}_{\mathrm{f}}} v_{\mathrm{f}} \phi_{\mathrm{f}} \mathrm{A}_{\mathrm{f}}=\sum_{\mathrm{f}}^{\mathrm{N}_{\mathrm{f}}} \Gamma_{\phi}\left(\nabla \phi_{\mathrm{f}}\right)_{\mathrm{n}} \mathrm{A}_{\mathrm{f}}+\mathrm{S}_{\phi} \mathrm{V}
$$

where $\mathrm{f}$ denotes face of the control volume; $\mathrm{N}_{\mathrm{f}}$ is number of faces enclosing cell.

After combing boundary conditions, equation (4) can be linearized and expressed in the computational domain as:

$$
\mathrm{a}_{\mathrm{p}} \phi=\sum_{\mathrm{nb}} \mathrm{a}_{\mathrm{nb}} \phi_{\mathrm{nb}}+\mathrm{b}
$$

where the subscript $\mathrm{nb}$ refers to neighbour cells; $\mathrm{a}_{\mathrm{p}}$ and $\mathrm{a}_{\mathrm{nb}}$ are the linearized coefficients for $\phi$ and $\phi_{\mathrm{nb}}$; b is the source term including Eq. (2).

The number of neighbours for each cell depends on the grid topology, but will typically equal the number of faces enclosing the cell. Similar equations can be written for each cell in the grid and this results in a set of algebraic equations to be solved using CFD techniques.

\subsection{Rules for distributor design}

The modified distributor was designed according to some rules proposed by this study. The rules are on the assumption that the plug flow is inviscid without circulation existing in channels of the distributor. The rules are made by satisfying the law of mass conservation and stated as follows:

1. Draw a radial line from the centre of the distributor surface to get a radius and evenly divide the radius to $\mathrm{N}$ segments (Method 1), or divide the radius to make rings, including the inner circle, with equal area (Method 2).

2. Make N-1 concentric circles on the surface to form N-1 rings and an inner circle in which the feed tube (inlet) is located. Calculate the areas and denote them $S_{1}$ to $S_{N}$ from inner to outer region on the distributor surface.

3. Determine $\mathrm{Mn}$ ( $\mathrm{n}=1$ to $\mathrm{N}-1$ ) uniformly spaced distribution openings surrounding each ring. Denote the opening area as An (No restrictions for the shape of the openings and an being different from ring to ring for Method 2).

4. Make sure opening-area density, $\left(\sum_{1}^{M n} A_{n}\right) / S_{n}$ on each ring $(n)$, including the inner circle, have the same value; it will keep the same total flow rate for each ring and the inner circle.

5. Denote the segment length of each channel (including its branch) from the inlet to the opening as $L_{j}$; then make sure total length $\left(\sum_{1}^{J} L_{j}\right)$ is the same for each channel on the 
surface. Besides, the cross-sectional area from the inlet to the opening along the channel is kept invariant to make flow rate conservation.

\subsection{Computer aided design and analysis}

\subsubsection{Solid modelling and mesh}

For most preparative and process scale chromatographic columns, DAC column ends include distributors (or collectors) and frits to distribute (or collect) fluid over the cross section of the column. Figure 2 displays illustrations of the DAC column with collector excluded. The engineering sketch, numerical model by SoldWorks 3D CAD and a mesh system for CFD computations are displayed in Fig. 2(a), 2(b) and 2(c), respectively [19]. The mesh is unstructured grid system with mixed hexahedra and tetrahedral grids. To effectively compute the present flow fields, the flow domain was split into smaller subdomains. We employed the mixed grid types to build the mesh around the distributor and frits. In the packed bed zone, structured hexahedral grids were used to fit the space inside the column. Moreover, finer grids were imposed along boundary walls to resolve the laminar boundary layer. Grid number for our parameter study was determined to be 0.8 million from grid independent tests and validated with experiments. The regions near wall, inlet and outlet were allocated a slightly higher mesh density as a velocity gradient was present. The desktop PC, $32 \mathrm{G}$ RAM with 4 cores $2.7 \mathrm{GHz}$ CPU, performed computation with iteration no. around 3000 and $3 \mathrm{hrs}$ computation for each case.

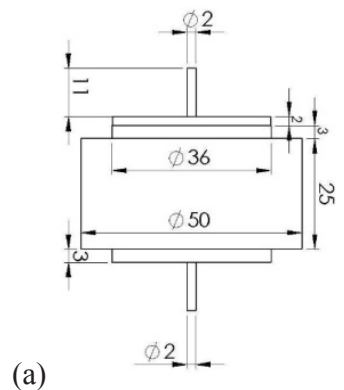

(b)
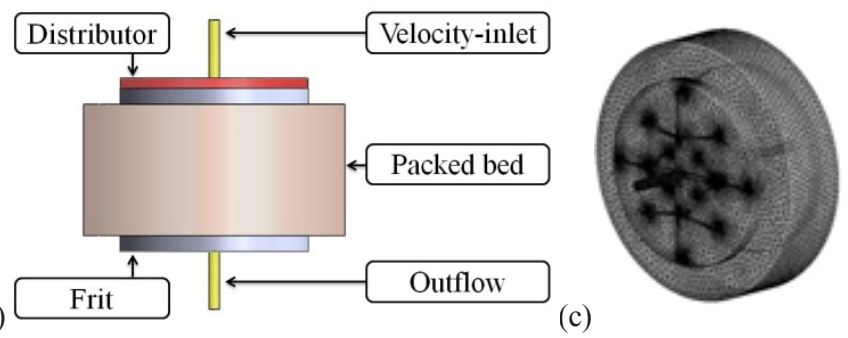

Fig. 2. Illustrations of the DAC column. (a) Engineering sketch. (b) Numerical solid modelling by CAD. (c) Mesh system for CFD computations.

\subsubsection{CFD for detailed flow fields}

The equations governing the present incompressible flow fields have no link between the continuity and momentum equations due to lack of the equation of state. To solve the set of algebraic equations derived from the governing equations, the SIMPLE algorithm was employed to carry out the calculations $[19,20]$. SIMPLE is an acronym for Semi-Implicit Method for Pressure Linked Equations. SIMPLE actually solves the pressure correction equation, which is derived from combining the discrete continuity and momentum equations. Therefore, the set of momentum and continuity equations are coupled and nonlinear so we solve the equations iteratively. The momentum equations are solved for the velocities; however, the newly obtained velocities don't satisfy continuity. Corrections to velocities and pressure are proposed to satisfy the discrete continuity equation in SIMPLE algorithm. After iterations for the above correction computations, the final solution can be obtained if the estimated residuals approach within required tolerances. 
In this study, the 3D numerical geometry model for the DAC column is created based from the engineering drawing as shown in Fig. 2(a)(b). Then we use CFD pre-processor, ANYSYS MESHING to transform the geometry model to the mesh system as shown in Fig. 2(c). After the mesh system is imported to the CFD FLUENT solver, numerical parameters, such as fluid properties, initial and boundary conditions have to be input. Besides, parameters for computation iterations and post processing for contour plotting need to be provided. According to the present numerical model, the velocity at inlet of the DAC column is set to be a constant value, which comes from experiments. The porous medium includes two zones, the frit part and the packed bed part as shown in Fig. 2(b). In fact, the collector at the rear portion of the DAC column is the same as the distributor in geometry. For the sake of limited computing resource, half of the DAC column can be adopted to study the header only and the outflow boundary condition are provided at outlet. The stationary solution of the momentum conservation was calculated first. After checking the convergent flow field, the concentration balances were switched on to make the transient simulation.

\section{Results and discussions}

Following the rules as stated previously, several new designs for distributor have been developed as shown in Fig. 3. Figure 3(a) illustrates the distributor with 3X4 circle openings located in a circle and 2 rings with equal area (Method 2). Figures 3(b) and 3(c) illustrate distributors with $3 \mathrm{X} 4$ circle openings and cross openings located in 3 circles, respectively, with same line segment radially (Method 1). In Fig. 3(c), $N$ is three; $M_{n}$ is four; $\mathrm{J}$ is two with open-area density equal to $8.83 \%$.

(a)

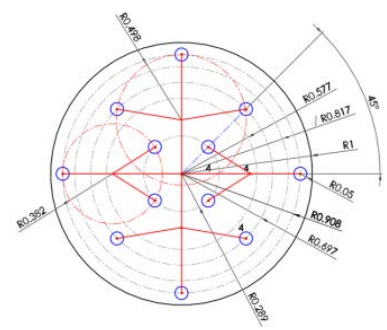

(b)

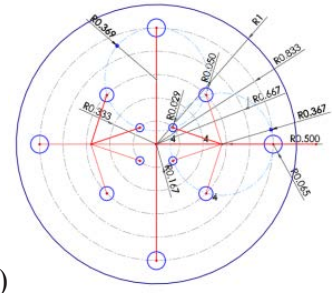

(c)

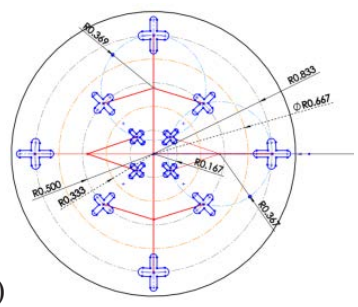

Fig. 3. Example models developed for the distributor by proposed rules (a) $3 X 4$ openings by Method 2. (b) $3 X 4$ circle openings by Method 1. (c) $3 X 4$ cross openings by Method 1.

\subsection{Validation experiment}

To show the feasibility of our proposed rules for distributor design, two models were used for experiments. The original distributor (commercial distributor) and the new designed one for experiments, including the solid modelling and the fabricated workpiese, are displayed in Figs. 4(a) and 4(b) and Figs. 5(a) and (b), respectively. The experiments were conducted at the Centre for Advanced Chromatographic Processing of I-Shou University. The working fluid is the solvent, ethanol, and the solute is $20 \mathrm{~mL}$ caffeine. The packed bed inside the column is full of $\mathrm{SiO}_{2}$ with 0.37 porosity and acts as the porous zone in computations. The UV detector detected signals with wavelength at $254 \mathrm{~nm}$ for caffeine. The experimental data were collected as an electronic signal, all values of which were divided by the area under the eluted pulse curve. Therefore, the obtained data can be regarded as the 
normalized concentration. Parameters for experiments are thereafter used for boundary conditions in CFD simulations.

To validate the flow profile improvement from the new distributor in DAC column, the number of theoretical units (NTU) is adopted. The concept of the number of theoretical units in chromatography has been developed to described and quantify the differences in the separating efficiency based on the eluted peak shape [5]. The efficiency of a DAC column is a statistical calculation of the standard deviation of the peak in unit time. It is measured in NTU or the height equivalent to a theoretical plate (HETP), which are defined as follows:

$$
\text { HETP }=\text { L / NTU }
$$

where L is the length of the packed bed and NTU stands for the number of theoretical units.

HETP is the preferred description of column efficiency as columns of any geometry can be compared as their efficiency per unit length. A higher NTU or a lower HETP indicates better column efficiency and it can be clearly demonstrated in Fig. 6, in which HETP from the new designed distributor are higher than that of the commercial one for two flow rates. However, experiments in Figures 6(a) and 6(b) show that better performances are obviously presented for longer length columns (high L/D) and poor performances for shorter length ones (low L/D) because there is no time for flow to distribute uniformly in a short column and the good distributor helps to overcome this drawback.

(a)

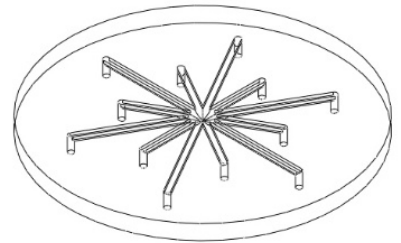

Fig. 4. The original distributor for experiments. (a) Solid modelling. (b) Fabricated workpiece.

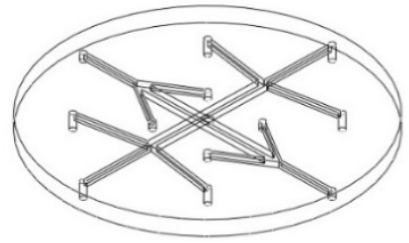

(b)

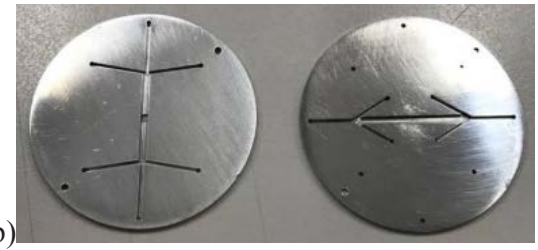

Fig. 5. The new designed distributor for experiments. (a) Solid modelling. (b) Fabricated workpiese.

\subsection{Numerical treatments for CFD}

The transient flow models were simulated based on the Finite Volume Method in ANSYS Fluent. The DAC column was initially filled with the solvent (ethanol) which remained in steady state. In addition, there was no free solute in ether the mobile phase or the stationary phase. After some elapsed time, the free solute mixture (caffeine) was introduced into the column using pulse injection. Furthermore, zero-gradient concentration was imposed on the wall and no-slip condition was applied for the eluent flow. Porous media are modelled by the addition of a source term to the standard momentum equations. This momentum source term contributes to the pressure gradient in the porous cell, creating a pressure drop that is 
proportional to the fluid velocity in the cell. In present numerical calculations, the rate of convergence slowed if the pressure drop was relatively large in the flow direction in a porous region. This slow convergence can occur because the momentum source term yields a loss of diagonal dominance in the matrix of discretized equations (Eq. (4)) solved. The best remedy for poor convergence of a problem involving a porous medium is to supply a good initial guess for the properties across the medium. Another possible way to deal with poor convergence in computation is to disable the porous media model temporarily and obtain an initial flow field. With the porous media model turned off, FLUENT will treat the porous zone as a fluid zone and calculate the flow field accordingly. Once an initial solution is obtained, or the calculation is proceeding steadily to convergence, we thus enable the porous media model and continue the calculation with the porous region included.

(a)

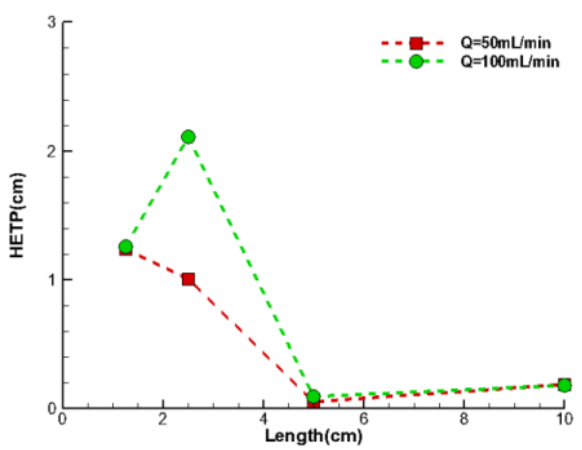

(b)

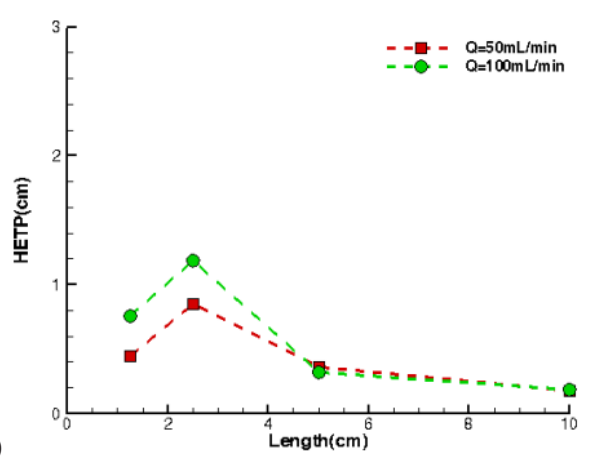

Fig. 6. Comparisons of HETP versus the column length for two flow rates. (a) The commercial distributor. (b) The new designed distributor.

Based on the Kozeny-Carman correlation in Eq. (7) and following the solution strategies as mentioned, we can obtain the convergent calculations and confirm numerical parameters for further simulation. The Kozeny-Carman correlation is represented by the following equation [9]:

$$
\alpha=\frac{\mathrm{d}_{\mathrm{p}}^{2} \cdot \varepsilon^{2}}{150(1-\varepsilon)^{2}}
$$

where $\alpha$ is permeability; $\varepsilon$ is porosity and $d_{p}$ is diameter of packing particles. The porosity is the volume fraction of fluid within the porous region (i.e., the open volume fraction of the medium). The porosity is set to be 1.0 (or permeability $\alpha \rightarrow \infty$; viscous resistance $1 / \alpha \rightarrow 0$ ) if the medium is treated as completely open. According to data from the manufacturer of the DAC column; $\mathrm{d}_{\mathrm{p}}=50 \times 10^{-6} \mathrm{~m} ; \varepsilon=0.37 ; \alpha=5.75 \times 10^{-12} \mathrm{~m}^{2}$. Therefore, viscous resistance is evaluated to be $1.74 \times 10^{11} \mathrm{~m}^{-2}$. Thus at the beginning of calculations, this value is a trial to seek the appropriate one which meets the experimental data within the required tolerance. In Fig. 7, the experimental datum was searched out via the CFD by the trial value as an initial input and the process is a kind of reverse engineering to obtain the values we expect. Figure 7 illustrates the relationship of pressure drop versus inlet flowrate for the DAC column in experiments and CFD calculations. Experimental and CFD data agree well and the curve reflects their linear property. Thus a linear function about the relationship of pressure drop versus inlet flow rate for the present DAC column can be proposed. In fact, in laminar flows through porous media, the pressure drop is typically proportional to velocity, which has been well known and described by the Darcy's law [7]. 


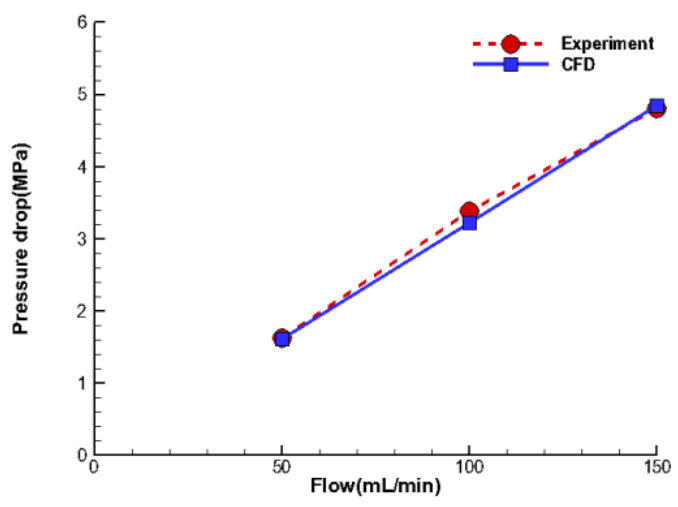

Fig. 7. Pressure drop versus inlet flow rate for the DAC column.

\subsection{Parameter analysis}

CFD is a good tool to describe the transient detailed flows by the time marching technique as shown in Fig. 8 (column length $\mathrm{L}=25 \mathrm{~mm}$; flow rate $\mathrm{Q}=100 \mathrm{~mL} / \mathrm{min}$ ). Figure 8 shows the mass fraction (concentration) contour evolution of the caffeine injected by a step function pattern after a period of time of achieving the steady-state flow field. The diameter of the tube connected to the column is much smaller than the column diameter. Without the distributor in the region near the inlet, the fluid particle near the axis will flow faster than that near the sidewall, which results in the curvature of the concentration profile. The wing of the curved profile may explain the peak tailing of the eluted peak because it will be eluted after the bulk part of the profile. Apparently, the distributor helps to forming plug flow at inlet (Figs. 8(a) and 8(b)) because of mass conservation and CFD helps to providing the visualization of concentration fields to see the distributor's contribution at various elapsed times (60 sec., $65 \mathrm{sec}$. and $70 \mathrm{sec}$.).

For comparing with the experimental data, the simulated chromatogram is the eluted curve of the area-weighted mass fraction average at outlet versus time. Besides, all simulated area-weighted values were normalized with dividing by the area, which was the integral of the eluted pulse curve. Figure 9 shows comparisons of experimental and simulated chromatograms for the original distributor in Fig. 9(a) and the new designed one in Fig. 9(b). Apparently, the experimental chromatogram of the new designed distributor appear to have higher peak and less trailing as compared with the original one, and the new distributor can provide better chromatography performance as shown in Fig. 6. However, simulated chromatograms are only minor distinct as compared by both distributors. It is possible that solid-phase properties were not realistically modelled and simulated. The flow and concentration profiles after the distributors were diffused in the same mechanism across the packed bed, and resulted in very similar chromatogram.

Before going further to study the pattern effect of the input solute on chromatograms, the different viscous resistances of the packed bed were simulated. The chromatogram with the homogeneous viscous resistance in porous zone is illustrated as the red line for comparisons in Fig. 10. In heterogeneous cases, the chromatogram of the green line for the small resistance in the radial direction shows the shorter retention time and the highest peak in Fig. 10(b). On the contrary, the chromatogram of the blue line for the small resistance in the axial direction shows the longer retention time and the lowest peak height. Viscous resistances are relevant to the flow speed and the ability to diffuse across the packed bed. Figure 11 shows the effect on the chromatogram for various injected patterns with the same time period. The eluted curves are similar in shape in Fig. 11(b) except the peak heights due 
to mass conservation of solute in Fig. 11(a). Besides, patterns of the injected solutes at the same time period seem to be less relevant to patterns of chromatograms. Figure 12 shows comparisons of chromatograms for the same quantity of solutes but different time periods. Four time periods of injected solute patterns are demonstrated in Fig. 12(a) and the respective chromatograms at outlet are displayed in Fig. 12(b). For the same quantity of inputs, the injected solutes of longer time period result in lower peak height, longer retention time and wide peak pulse in chromatograms.

(a)

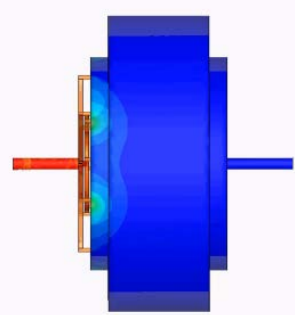

(b)

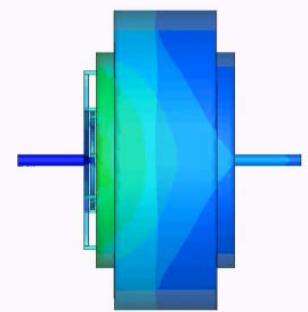

(c)

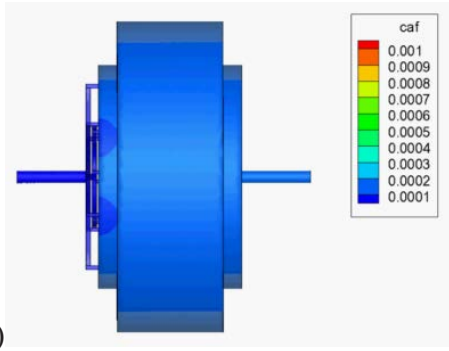

Fig. 8. Concentration contours of caffeine at various times (a) $60 \mathrm{sec}$. (b) $65 \mathrm{sec}$. (c) $70 \mathrm{sec}$.

(a)

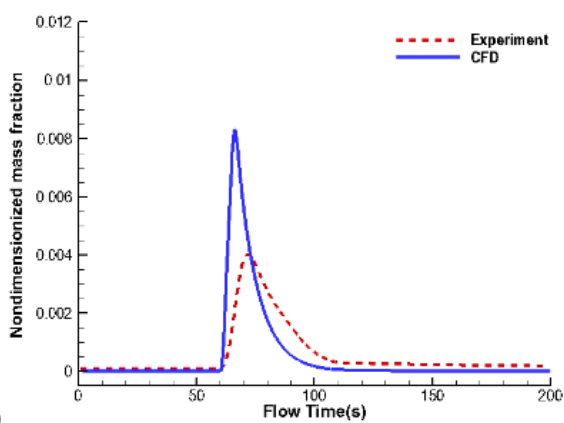

(b)

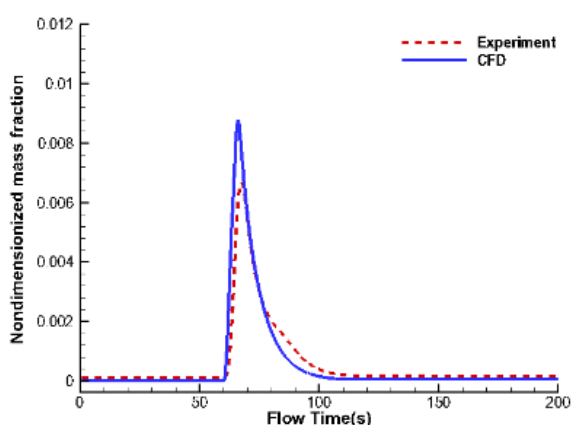

Fig. 9. Comparisons of experimental and simulated chromatograms at outlet. (a) For the original distributor. (b) For the new designed distributor.

(a)

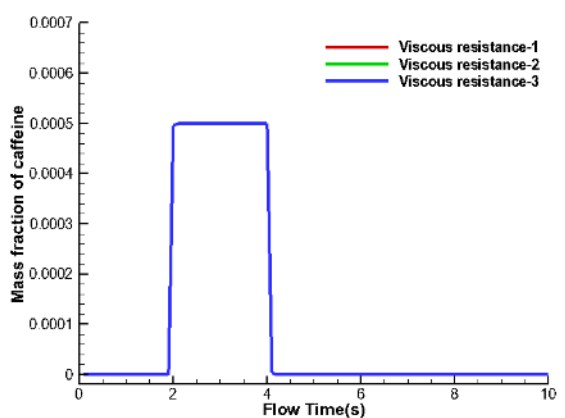

(b)

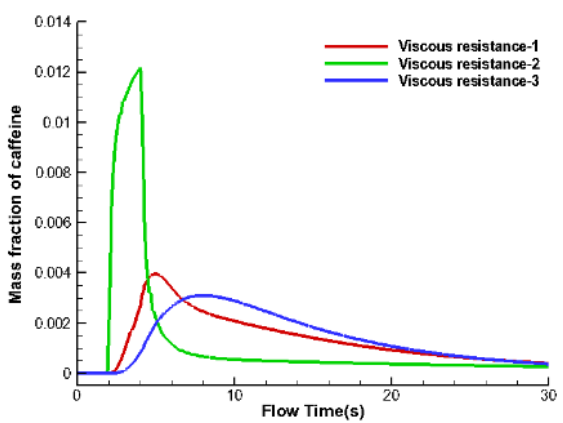

Fig. 10. Comparisons of chromatograms for different viscous resistances. (a) Three solutes of same quantity and patterns. (b) The chromatograms at outlet. 
(a)

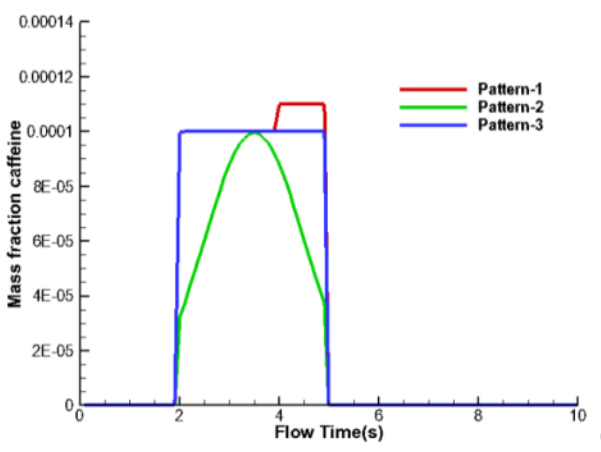

(b)

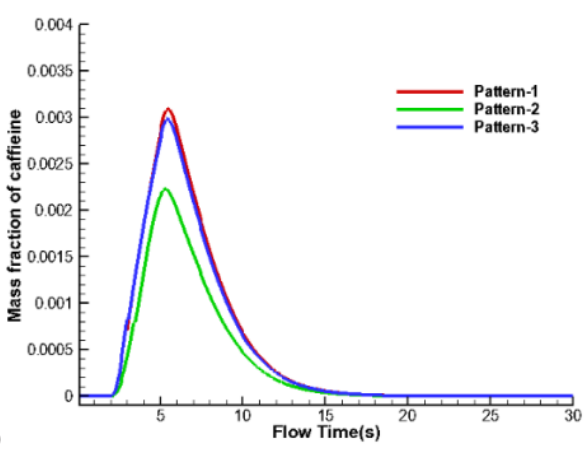

Fig. 11. Comparisons of chromatograms for different injected solute patterns. (a) Three injected solute patterns. (b) The chromatograms at outlet.

(a)

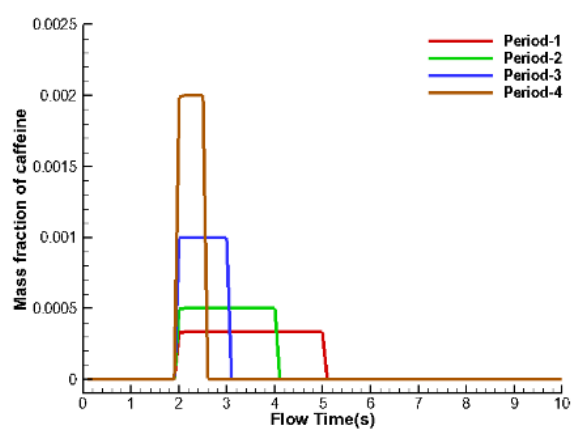

(b)

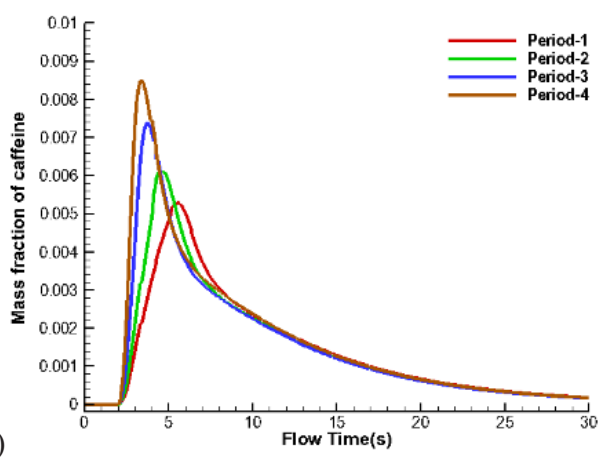

Fig. 12. Comparisons of chromatograms for same quantity of solutes but different time periods. (a) Different injected patterns of solutes. (b) The chromatograms at outlet.

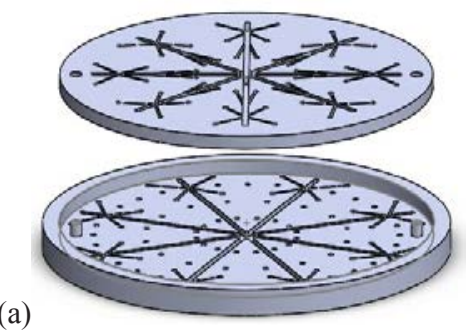

(b)
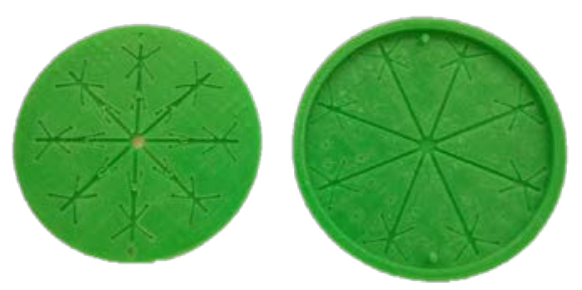

Fig. 13. The new compound distributor. (a) Solid modelling. (b) Prototypes by $3 \mathrm{D}$ printing.

\section{Concluding remarks}

The rules for designing distributors are proposed for DAC columns in this study. The new distributor was developed based on the proposed rules and validated by experiments, which shows the new distributor performs better than the original one. In addition, the CAD and CFD techniques were used to build solid modelling and thereafter conduct numerical analysis, which can provide detailed numerical flows and visualizations. CFD analysis show that there exist the longer retention time and the lowest peak height in chromatograms for small viscous resistance in the axial direction in heterogeneous beds. Moreover, patterns of the injected solutes at the same time period seem to be less relevant to patterns of chromatograms due to highly diffusive property in packed beds. For the same quantity of inputs, injected solutes of longer time period result in lower peak height, longer retention 
time and wide peak pulse in chromatograms. Based on present computer aided techniques, the new compound distributor has been designed, 3D printed and processed in factory for testing, evaluation and practical chromatographic applications. It is anticipated that this distributor can perform better column efficiency especially for DAC columns with low L/D.

\section{Acknowledgements}

Financial Assistance for this research provided by the Taiwanese Ministry of Science and Technology, under contract number MOST 106-2218-E-214-001, is greatly appreciated.

\section{References}

1. G. Guiochon, J. Chromatography. A 965, 129 (2002)

2. J.Y. Song, K.M. Kim, C.H. Lee, J. Chromatography. A 1471, 102 (2016)

3. M.T. Liang, R.C. Liang, J. Chromatography. A 1229, 107 (2012)

4. M.T. Liang, et al., The Journal of Supercritical Fluids. 79, 324 (2013)

5. S. Tan, B.C. Khoo, MIT Libraries. https://dspace.mit.edu/handle/1721.1/3700 (2003)

6. Q.S. Yuan, et al., J. Chromatography. A 831, 149 (1999)

7. Y. Wu, C. Ching, Chromatographia. 57, 329 (2003)

8. J.C. Park, K. Raghavan, S.J. Gibbs, J. Chromatography. A 945, 65 (2002)

9. Y. Li, C.W. Park, Industrial \& engineering chemistry research. 37, 2005 (1998)

10. F.G. Lode, et al., J. Chromatography. A 796, 3 (1998)

11. C.C. Allen, N.C. Durham, Fluid distributor. (United States Patent, US 4537217, 1985)

12. K. Gebauer, J. Kranse, M. Lundkvist, Fluid distributor unit. (United States Patent, US 2012/0074051, 2012)

13. W. Shyy, Computational modeling for fluid flow and interfacial transport. (Courier Corporation, 2014)

14. W. Choopakdee, et al., in Computer Aided Chemical Engineering. edited by P.S. Varbanov, J. J. Klemeš, L. P. Yen, 33, 1393 (2014)

15. L.F. Gomes, et al., Chemical Engineering Science. 132, 46 (2015)

16. L.F. Gomes, et al., Chemical Engineering Science. 153, 188 (2016)

17. Y. Cohen, A. Metzner, AIChE Journal. 27, 705 (1981)

18. Y.N. Jeng, J.L. Chen, Numerical Heat Transfer, Part B: Fundamentals. 22, 211 (1992)

19. ANSYS FLUENT 13 Theory Guide. (ANSYS, Inc. USA, 2010)

20. S.V. Patankar, Numerical heat transfer and fluid flow. (Hemisphere Pub. Co New York, 1980) 